\title{
Processing of Azimuth-Invariant Bistatic SAR Data Using the Range Doppler Algorithm
}

\author{
Yew Lam Neo, Frank H. Wong, and Ian G. Cumming, Life Senior Member, IEEE
}

\begin{abstract}
This paper discusses bistatic synthetic aperture radar processing (complex image formation) using the Range Doppler Algorithm. The key step is to use an analytical form of the signal spectrum derived by the method of series reversion. The spectrum is used for secondary range compression (SRC), range cell migration correction, and azimuth compression. The algorithm is able to focus the azimuth-invariant bistatic configuration where the transmitter and receiver platforms are moving in parallel tracks with identical velocities. Moreover, the algorithm is able to handle reasonably high squints and wide apertures because SRC can be performed in the 2-D frequency domain.
\end{abstract}

Index Terms-Bistatic synthetic aperture radar (SAR) processing, range cell migration correction (RCMC), Range Doppler Algorithm (RDA), secondary range compression (SRC), series reversion.

\section{INTRODUCTION}

$\mathbf{R}$ ECENT interest has arisen in processing bistatic synthetic aperture radar (SAR) data partly because of two airborne experiments that have been performed in the last few years [1], [2]. Bistatic SAR range histories, unlike monostatic ones, are azimuth variant in general as both the transmitter and the receiver can assume different motion trajectories. Nevertheless, the bistatic system can remain azimuth invariant by restricting the transmitter and receiver platform motions to follow parallel tracks with identical velocities. In this case, the baseline between the two platforms does not vary with time. ${ }^{1}$

This azimuth-invariant property is important to conventional monostatic algorithms such as the Range Doppler Algorithm (RDA) [3]-[5] and Chirp Scaling Algorithm (CSA) [6], or any algorithm that takes advantage of block processing in the azimuth. This is because the processing efficiency is achieved by taking advantage of the fact that point targets with the same range of closest approach collapse to the same range history in the range Doppler domain. Performing one range cell migration correction (RCMC) operation in this domain achieves the correction of a whole family of targets. Moreover,

Manuscript received April 12, 2007.

Y. L. Neo is with DSO National Laboratories, Singapore 118230 (e-mail: nyewlam@dso.org.sg).

F. H. Wong is with MacDonald Dettwiler and Associates, Richmond, BC V6V 2J3, Canada.

I. G. Cumming is with the Department of Electrical and Computer Engineering, University of British Columbia, Vancouver, BC V6T 1Z4, Canada.

Color versions of one or more of the figures in this paper are available online at http://ieeexplore.iee.org.

Digital Object Identifier 10.1109/TGRS.2007.909090

${ }^{1}$ The baseline is the vector connecting the two platforms. The azimuth invariant is equivalent to a fixed baseline. Each beam can have different squint and incidence angles. The bistatic angle is the angle $\beta$ subtended by the baseline at the target (see Fig. 3). the range Doppler domain allows the azimuth compression parameters to be conveniently changed with range. The purpose of this paper is to examine whether the same advantages can be obtained in the bistatic case when the data are azimuth invariant.

The processing of monostatic strip-map SAR data, which are acquired with the platform following a straight line flight path, is based on a hyperbolic range equation. However, as the transmitter and receiver ranges are independent in the bistatic case, a double hyperbolic range equation results, which leads to a double square root in the range equation. The sum of the two square roots is referred to as the "flat-top" hyperbola [7]-[9]. As monostatic SAR processing algorithms assume the (single) hyperbolic range equation, they must be modified to handle bistatic SAR data.

For small baseline/range ratios, the range equation is nearly hyperbolic, and conventional monostatic algorithms can be used to focus the data without significantly affecting the image quality. However, when the separation between the transmitter and the receiver increases, the bistatic range equation diverges further from the hyperbolic form. Modifications to monostatic algorithms are necessary to allow them to focus with this different geometry model.

The main difficulty in modifying monostatic algorithms to handle the azimuth-invariant bistatic configuration lies in finding an analytical solution for the 2-D target spectrum [8] (the double square root function makes it hard to apply the principle of stationary phase). Several algorithms have been developed to overcome this difficulty.

Modified $\omega-k$ algorithms were introduced in [10] and [11]. These algorithms work in the 2-D frequency domain (2-D FD) and make use of numerical methods to calculate the double square root phase term. Bamler and Boerner [12] proposed a focusing algorithm that replaces the analytical SAR transfer functions with numerical equivalents. Their algorithm is able to handle the azimuth-invariant case including squint. A preprocessing technique derived from "Dip and Move Out" in the seismic literature [13] is used to transform the bistatic data to a monostatic equivalent [7]. The data can subsequently be focused with any monostatic algorithm. Loffeld et al. [9] derived an approximate bistatic point target spectrum, and a few subsequent algorithms were developed based on this approach [14], [15]. In particular, Rodríguez-Cassolá et al. [16] made use of this point target spectrum to develop a bistatic RDA and a bistatic CSA.

While all these algorithms are able to handle azimuthinvariant cases, the bistatic geometries that can be focused by these algorithms are often limited by the accuracy of the point target spectrum. Recently, an accurate 2-D point target spectrum has been derived based on the reversion of a power series for the general bistatic case [17]. Our approach is to apply 
this spectral result in a modified RDA so that it can handle the azimuth-invariant case with improved accuracy.

Note that the conventional RDA does not normally do any processing in the 2-D FD. Secondary range compression (SRC) is commonly applied in the azimuth time domain as part of the range compression operation [18]. This approximation limits the degree of squint and the extent of the aperture that can be accurately processed. Focusing high squint and wide aperture cases is not a trivial task, as processing is complicated by the range/Doppler coupling in the received data, which degrades the focusing ability of the conventional RDA. The squint-aperture cases that the RDA can accurately handle are considerably extended when SRC is performed in the 2-D FD, since SRC takes on an increasing amount of azimuth frequency dependence as the squint or aperture increases (refer to SRC Option 2 in [19]). The 2-D FD operations come at the expense of computing time and are therefore avoided if possible.

This paper begins with a brief description of the bistatic signal model and a derivation of its 2 -D point target spectrum in Section II, where an L-band example is given. The operations in the modified RDA are outlined in Section III, and the important 2-D FD phase equations are derived. A C-band airborne radar simulation is used to demonstrate the accuracy of the algorithm in Section IV. In Section V, an efficient way to combine the SRC with range compression is developed for certain squinted moderate aperture cases.

\section{2-D Spectrum OF A Bistatic SignAL}

The RDA developed in this paper is based on a 2-D spectrum of the reference point target that is derived in [17] and repeated here for convenience as

$$
S\left(f_{\tau}, f_{\eta}\right)=W_{r}\left(f_{\tau}\right) W_{\mathrm{az}}\left(f_{\eta}+\left(f_{o}+f_{\tau}\right) \frac{k_{1}}{c}\right) \exp \left\{j \phi\left(f_{\tau}, f_{\eta}\right)\right\}
$$

where the phase function is given by

$$
\begin{aligned}
\phi\left(f_{\tau}, f_{\eta}\right)= & -2 \pi\left(\frac{f_{o}+f_{\tau}}{c}\right) R_{\text {cen }}-\frac{\pi f_{\tau}^{2}}{K_{r}} \\
& +2 \pi \frac{c}{4 k_{2}\left(f_{o}+f_{\tau}\right)}\left(f_{\eta}+\left(f_{o}+f_{\tau}\right) \frac{k_{1}}{c}\right)^{2} \\
& +2 \pi \frac{c^{2} k_{3}}{8 k_{2}^{3}\left(f_{o}+f_{\tau}\right)^{2}}\left(f_{\eta}+\left(f_{o}+f_{\tau}\right) \frac{k_{1}}{c}\right)^{3} \\
& +2 \pi \frac{c^{3}\left(9 k_{3}^{2}-4 k_{2} k_{4}\right)}{64 k_{2}^{5}\left(f_{o}+f_{\tau}\right)^{3}}\left(f_{\eta}+\left(f_{o}+f_{\tau}\right) \frac{k_{1}}{c}\right)^{4} \\
& +\cdots
\end{aligned}
$$

where $K_{r}$ is the FM rate of the range pulse, $c$ is the speed of light, and $f_{o}$ is the center frequency of the transmitted pulse. $W_{r}($.$) represents the spectral shape of the transmitted pulse,$ and $W_{\mathrm{az}}($.$) represents the shape of the Doppler spectrum. f_{\tau}$ is the range frequency, and $f_{\eta}$ is the azimuth frequency. The definition of the bistatic range at the aperture center $R_{\text {cen }}$ and the derivatives $\left(k_{1}, k_{2}, k_{3}\right.$, and $\left.k_{4}\right)$ of the expanded version of the range equation in azimuth time $\eta$ are given in [17]. These derivatives are weakly range dependent.

The accuracy of the spectrum is only limited by the number of terms used in the expansion of (2). For a higher resolution

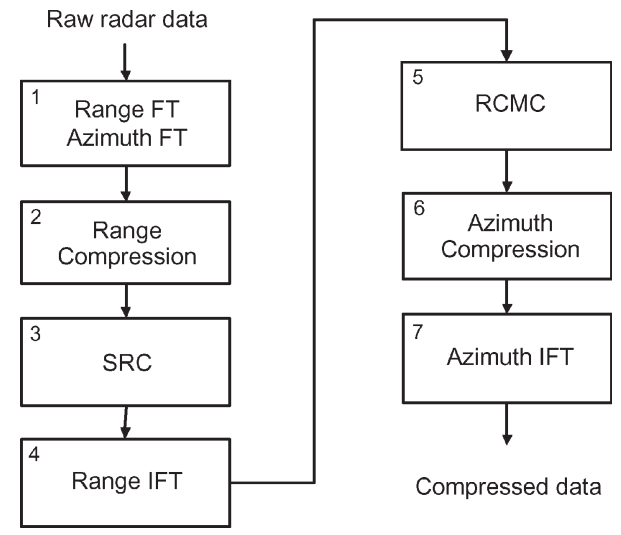

Fig. 1. Functional block diagram of the bistatic RDA.

or a wider aperture, we may need to include more phase terms to avoid significant degradation in the focusing algorithm. An example of the convergence of the series is given in [17].

In [20], Eldhuset inverted a fourth-order range equation to give an "exact" transfer function for a spaceborne monostatic SAR. This method has been extended in [21] for an bistatic azimuth-invariant spaceborne SAR. The method derived here is more general and can handle higher squints and wider apertures by adding more terms in the phase equation.

The point target spectrum (1) is central to the bistatic RDA that we discuss in this paper. In Section III, we show how this point target spectrum can be used to process the raw bistatic SAR data for the azimuth-invariant case.

\section{BISTATIC RDA}

The processing steps of the bistatic RDA are shown in Fig. 1, where four Fourier transforms (FT) are used. They are the same as the RDA when SRC is applied in the 2-D FD except that the new spectrum is used in the SRC, RCMC, and azimuth compression operations. Note that range compression and SRC are both phase multiplies and can be combined for efficiency. RCMC is applied with an interpolator, and azimuth compression is applied with a range-dependent phase multiply.

\section{A. Analytical Development}

The development of the bistatic RDA begins with the 2-D spectrum (2) of the point target being considered. The first step is to replace the $1 /\left(f_{\tau}+f_{o}\right)$ terms in (2) with the following power series expansions:

$$
\begin{aligned}
& \frac{1}{\left(f_{o}+f_{\tau}\right)}=\frac{1}{f_{o}}\left[1-\frac{f_{\tau}}{f_{o}}+\left(\frac{f_{\tau}}{f_{o}}\right)^{2}+\cdots\right] \\
& \frac{1}{\left(f_{o}+f_{\tau}\right)^{2}}=\frac{1}{f_{o}^{2}}\left[1-\frac{2 f_{\tau}}{f_{o}}+3\left(\frac{f_{\tau}}{f_{o}}\right)^{2}+\cdots\right] \\
& \frac{1}{\left(f_{o}+f_{\tau}\right)^{3}}=\frac{1}{f_{o}^{3}}\left[1-\frac{3 f_{\tau}}{f_{o}}+6\left(\frac{f_{\tau}}{f_{o}}\right)^{2}+\cdots\right] .
\end{aligned}
$$

These power series quickly converge because $f_{o} \gg\left|f_{\tau}\right|$ in practice. Substituting (3)-(5) into (2), an explicit form of the 
phase of the 2-D spectrum can be obtained. The phase term in (2) can be decomposed into the following components:

$$
\begin{aligned}
\phi\left(f_{\tau}, f_{\eta}\right) \approx \phi_{\mathrm{rg}}\left(f_{\tau}\right) & +\phi_{\mathrm{az}}\left(f_{\eta}\right) \\
& +\phi_{\mathrm{rcm}}\left(f_{\tau}, f_{\eta}\right)+\phi_{\mathrm{src}}\left(f_{\tau}, f_{\eta}\right)+\phi_{\mathrm{res}} .
\end{aligned}
$$

Each of these phase terms can be interpreted as follows:

- The first phase term $\phi_{\mathrm{rg}}$ represents the range modulation as

$$
\phi_{\mathrm{rg}}\left(f_{\tau}\right)=-\frac{\pi f_{\tau}^{2}}{K_{r}} .
$$

This phase term is dependent only on $f_{\tau}$ and thus can be separated from the other phase terms. Range compression uses a phase multiply to remove this phase term. Alternatively, the data could be range compressed in the range frequency azimuth time domain just after the range FT. This operation is the same as in the monostatic case as the bistatic geometry has no direct effect on the pulse modulation.

- The second phase term $\phi_{\mathrm{az}}$ represents the azimuth modulation. It is solely dependent on $f_{\eta}$ and will be removed by the azimuth-matched filtering as

$$
\begin{aligned}
\phi_{\mathrm{az}}\left(f_{\eta}\right) \approx 2 \pi\{ & \frac{1}{4 k_{2}}\left[2 k_{1} f_{\eta}+\frac{c}{f_{o}} f_{\eta}^{2}\right] \\
+ & \frac{k_{3}}{8 k_{2}^{3}}\left[3 k_{1}^{2} f_{\eta}+\frac{3 k_{1} c}{f_{o}} f_{\eta}^{2}+\frac{c^{2}}{f_{o}^{2}} f_{\eta}^{3}\right] \\
+ & \frac{9 k_{3}^{2}-4 k_{2} k_{4}}{64 k_{2}^{5}}\left[4 k_{1}^{3} f_{\eta}+\frac{6 k_{1}^{2} c}{f_{o}} f_{\eta}^{2}\right. \\
& \left.\left.+\frac{4 k_{1} c^{2}}{f_{o}^{2}} f_{\eta}^{3}+\frac{c^{3}}{f_{o}^{3}} f_{\eta}^{4}\right]\right\} .
\end{aligned}
$$

Because of the significant range dependence of these terms, the azimuth compression is applied in the range Doppler domain.

- The third phase term $\phi_{\mathrm{rcm}}$ is linearly dependent on the range frequency $f_{\tau}$ and represents the range cell migration term as

$$
\begin{aligned}
& \phi_{\mathrm{rcm}}\left(f_{\tau}, f_{\eta}\right) \\
& \begin{aligned}
& \approx 2 \pi f_{\tau}\{-\frac{R_{\mathrm{cen}}}{c}+\frac{1}{4 k_{2}}\left[\frac{k_{1}^{2}}{c}-\frac{c}{f_{o}^{2}} f_{\eta}^{2}\right] \\
&+\frac{k_{3}}{8 k_{2}^{3}}\left[\frac{k_{1}^{3}}{c}-\frac{3 k_{1} c}{f_{o}^{2}} f_{\eta}^{2}-\frac{2 c^{2}}{f_{o}^{3}} f_{\eta}^{3}\right] \\
&+\frac{9 k_{3}^{2}-4 k_{2} k_{4}}{64 k_{2}^{5}}\left[\frac{k_{1}^{4}}{c}-\frac{6 k_{1}^{2} c}{f_{o}^{2}} f_{\eta}^{2}-\frac{8 k_{1} c^{2}}{f_{o}^{3}} f_{\eta}^{3}\right. \\
&\left.\left.-\frac{3 c^{3}}{f_{o}^{4}} f_{\eta}^{4}\right]\right\} .
\end{aligned}
\end{aligned}
$$

Note that the terms inside the large braces represent the range cell migration (RCM) displacement. As the $k$ coefficients depend on range, as does the $R_{\text {cen }}$ term, this range displacement must be compensated in the range Doppler domain. This is allowed since there is no range frequency dependence in these terms. The displacement is corrected using a range direction interpolator, as in the monostatic RDA.

- The fourth phase term $\phi_{\mathrm{src}}$ represents the range/azimuth coupling term as

$$
\begin{aligned}
\phi_{\mathrm{src}}\left(f_{\tau}, f_{\eta}\right) \approx 2 \pi\left\{\frac{c}{4 k_{2} f_{o}}\left[\left(\frac{f_{\tau}}{f_{o}}\right)^{2}-\left(\frac{f_{\tau}}{f_{o}}\right)^{3}\right] f_{\eta}^{2}\right. \\
+\frac{k_{3}}{8 k_{2}^{3}}\left[3 k _ { 1 } ( \frac { c } { f _ { o } } ) \left(\left(\frac{f_{\tau}}{f_{o}}\right)^{2}\right.\right. \\
\left.\quad-\left(\frac{f_{\tau}}{f_{o}}\right)^{3}\right) f_{\eta}^{2}+\left(\frac{c}{f_{o}}\right)^{2}\left(3\left(\frac{f_{\tau}}{f_{o}}\right)^{2}\right. \\
\left.\left.\quad-4\left(\frac{f_{\tau}}{f_{o}}\right)^{3}\right) f_{\eta}^{3}\right]+\frac{9 k_{3}^{2}-4 k_{2} k_{4}}{64 k_{2}^{5}} \\
\times\left[6 k_{1}^{2}\left(\frac{c}{f_{o}}\right)\left(\left(\frac{f_{\tau}}{f_{o}}\right)^{2}-\left(\frac{f_{\tau}}{f_{o}}\right)^{3}\right) f_{\eta}^{2}\right. \\
+4 k_{1}\left(\frac{c}{f_{\mathrm{o}}}\right)^{2}\left(3\left(\frac{f_{\tau}}{f_{o}}\right)^{2}-4\left(\frac{f_{\tau}}{f_{o}}\right)^{3}\right) f_{\eta}^{3} \\
\left.\left.+\left(\frac{c}{f_{o}}\right)^{3}\left(6\left(\frac{f_{\tau}}{f_{o}}\right)^{2}-10\left(\frac{f_{\tau}}{f_{\mathrm{o}}}\right)^{3}\right) f_{\eta}^{4}\right]\right\} .
\end{aligned}
$$

This phase term is the remaining contribution that depends on $f_{\eta}$ and $f_{\tau}$. This phase term becomes significant in higher squint, finer resolution, and longer wavelength cases. If uncompensated, the range/azimuth coupling may cause significant degradation in the resolution, especially in the range direction. The SRC is used to remove this coupling term. SRC is applied in the 2-D FD domain, as the strongest dependencies exist in this domain. However, the SRC term is weakly range dependent, and (10) must be evaluated at a specific range called the reference range, which is usually at the swath center. While the SRC that corrects the phase at the scene center is often sufficient for the whole scene, for wider range swaths, it may be necessary to segment the scene into range invariance regions whose width is selected by the quadratic phase error allowed.

- The last phase term $\phi_{\text {res }}$ represents a residual phase as

$$
\begin{aligned}
\phi_{\text {res }} \approx 2 \pi\{ & -\frac{f_{o}}{c} R_{\text {cen }}+\frac{1}{4 k_{2}}\left[\frac{k_{1}^{2}}{c} f_{o}\right] \\
& \left.+\frac{k_{3}}{8 k_{2}^{3}}\left[\frac{k_{1}^{3}}{c} f_{o}\right]+\frac{9 k_{3}^{2}-4 k_{2} k_{4}}{64 k_{2}^{5}}\left[\frac{k_{1}^{4}}{c} f_{o}\right]\right\} .
\end{aligned}
$$

As this phase does not depend on the range frequency or azimuth frequency, it has no effect on the focusing process. However, it does depend on the target range and should only be ignored if a magnitude image is the final product.

After removing the range modulation and range/azimuth coupling using the phase terms (7) and (10) in the 2-D FD, 
(a)

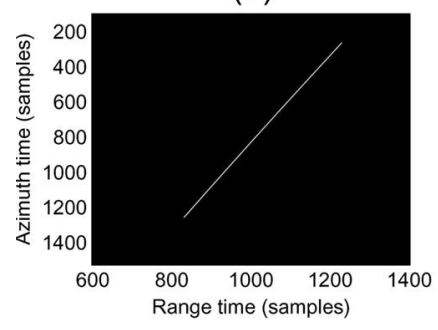

(c)

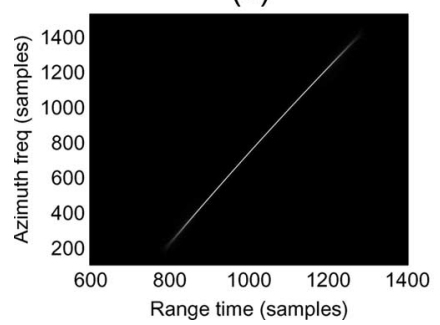

(b)

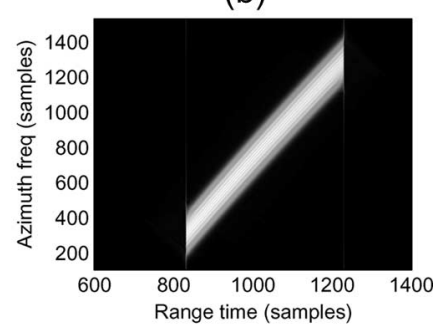

(d)

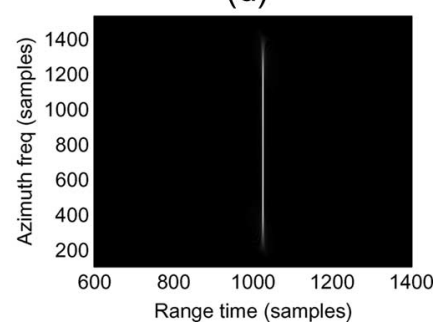

Fig. 2. How the target trajectories are changed by SRC and RCMC. (a) Range compressed. (b) Data after azimuth FT. (c) Data after SRC. (d) Data after RCMC.

an inverse range FT is performed to obtain a 2-D signal in the range Doppler domain as

$S_{\mathrm{rd}}\left(\tau, f_{\eta}\right)=\widetilde{\rho}_{r}\left(\tau-\frac{\varphi_{\mathrm{rcm}}}{c}, f_{\eta}\right) \widetilde{W}_{\mathrm{az}}\left(f_{\eta}-f_{\eta_{c}}\right) \exp \left\{-j \phi_{\mathrm{az}}\left(f_{\eta}\right)\right\}$

where the range envelope $\widetilde{\rho}_{r}\left(\tau, f_{\eta}\right)$ has a sinc-like shape in range, and the range migration term $\varphi_{\mathrm{rcm}}$ is related to the phase $\phi_{\mathrm{rcm}}\left(f_{\eta}\right)$ by

$$
\phi_{\mathrm{rcm}}\left(f_{\tau}, f_{\eta}\right)=-2 \pi f_{\tau} \frac{\varphi_{\mathrm{rcm}}\left(f_{\eta}\right)}{c} .
$$

The range inverse FT turns the linear phase component in $f_{\tau}$ into the time delay $\varphi_{\mathrm{rcm}} / c$ appearing in (12). The function $\widetilde{W}_{\mathrm{az}}\left(f_{\eta}-f_{\eta_{c}}\right)$ is the target envelope in the Doppler frequency domain, where the average Doppler frequency (the Doppler centroid) of the target is given by

$$
f_{\eta_{c}}=-f_{o} \frac{k_{1}}{c}
$$

RCMC is performed by a range-varying interpolation operation at this stage. The RCMC operation straightens the trajectories so that they now run parallel to the azimuth frequency axis. The final step is azimuth compression, which focuses each target to its mid-aperture range and mid-aperture time. The focused point target signal is given by

$$
\hat{s}(\tau, \eta)=\rho_{r}\left(\tau-\frac{k_{1}}{c} \eta\right) \rho_{\mathrm{az}}(\eta) \exp \left\{j 2 \pi f_{\eta_{c}} \eta\right\}
$$

where $\rho_{r}(\cdot)$ and $\rho_{\mathrm{az}}(\cdot)$ are the sinc-like compressed pulse envelopes. The exponential term in (15) is a linear phase ramp that occurs when the beams are squinted.

\section{B. Compression Example}

The effect of these terms and their compensation is illustrated in Fig. 2 using an example of a squinted bistatic SAR. The same

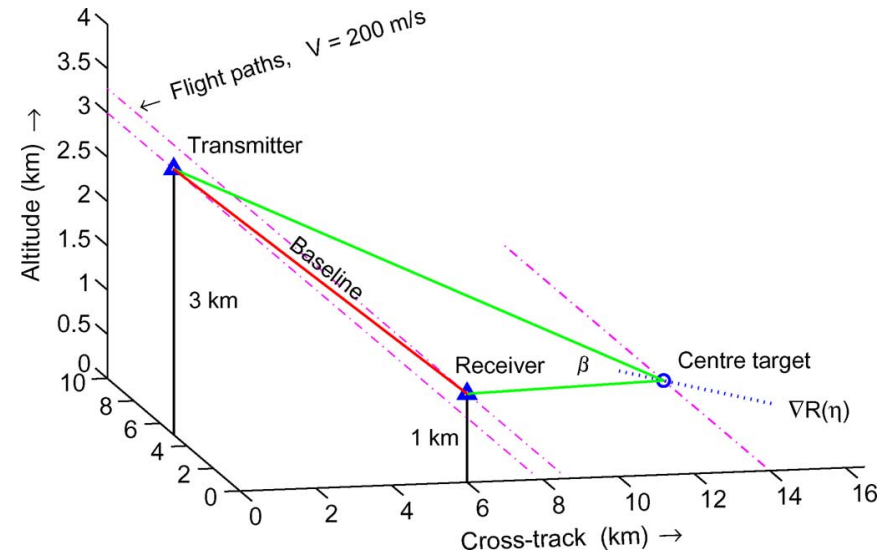

Fig. 3. Geometry of the bistatic simulation example (3-D view).

parameters are used as in the C-band simulation described in Section IV except that only a single point target is used in the present section.

The range-compressed target trajectory has a large migration of several hundred range cells, as shown in Fig. 2(a). When transformed into the range Doppler domain, the range/azimuth coupling causes the trajectory to be dispersed in range by 100 range cells (about 50 range resolution elements) [see Panel (b)]. SRC removes the range/azimuth coupling effect, as shown in Fig. 2(c) after the range inverse FT. We can view this process as the recompression of data in range, hence the name "SRC."

After RCMC, the majority of the energy from the point target falls into one range cell, as shown in Fig. 2(d). Without SRC, the energy would remain spread over many range cells, which results in significant resolution degradation, as in the monostatic case.

\section{Application to the CSA}

The CSA [6] is an alternate way of implementing an RDAlike algorithm. It differs in that RCMC is performed in two parts (i.e., a differential and a bulk part), and the interpolation operation is avoided. First, a phase multiply is applied to the rangeuncompressed data in the range Doppler domain to equalize the target migration at different ranges. Then, a bulk RCMC is done in the 2-D FD to complete the RCMC. Range compression and SRC are also performed during the same phase multiply for efficiency.

Because of the 2-D FD operations of the CSA, it is straightforward to apply the bistatic range compression and SRC phase terms (7) and (10) to the CSA. For the RCMC operation, the bulk component is (9) with the range set to a mid-swath reference range and applied in the same 2-D FD. The differential component is computed as the difference between (9) evaluated at the explicit range and that at the reference range. The differential component is inverse FTed and applied in the range Doppler domain, as in the monostatic case [6], [19].

\section{Bistatic Simulation Example}

To prove the validity of the formulation and to show how a whole scene can be focused, a simulation based on a flat earth model is presented in this section. The radar geometry is illustrated in Fig. 3. 
TABLE I

SIMULATION PARAMETERS

\begin{tabular}{|c|c|c|}
\hline Simulation parameters & Transmitter & Receiver \\
\hline \hline Velocity in x direction & $0 \mathrm{~m} / \mathrm{sec}$ & $0 \mathrm{~m} / \mathrm{sec}$ \\
\hline Velocity in y direction & $200 \mathrm{~m} / \mathrm{sec}$ & $200 \mathrm{~m} / \mathrm{sec}$ \\
\hline Velocity in z direction & $0 \mathrm{~m} / \mathrm{sec}$ & $0 \mathrm{~m} / \mathrm{sec}$ \\
\hline Center frequency & \multicolumn{2}{|c|}{$5.3 \mathrm{GHz}$} \\
\hline Range bandwidth & \multicolumn{2}{|c|}{$80 \mathrm{MHz}$} \\
\hline Doppler bandwidth & \multicolumn{2}{|c|}{$194 \mathrm{~Hz}$} \\
\hline Altitude & $3000 \mathrm{~m}$ & $1000 \mathrm{~m}$ \\
\hline Nominal target range & $15237 \mathrm{~m}$ & $13010 \mathrm{~m}$ \\
\hline Nominal squint angle & $20^{\circ}$ & $51.7^{\circ}$ \\
\hline Nominal bistatic angle & \multicolumn{2}{|c|}{$31.9^{\circ}$} \\
\hline Baseline distance between platforms & \multicolumn{2}{|c|}{$8062 \mathrm{~m}$} \\
\hline
\end{tabular}

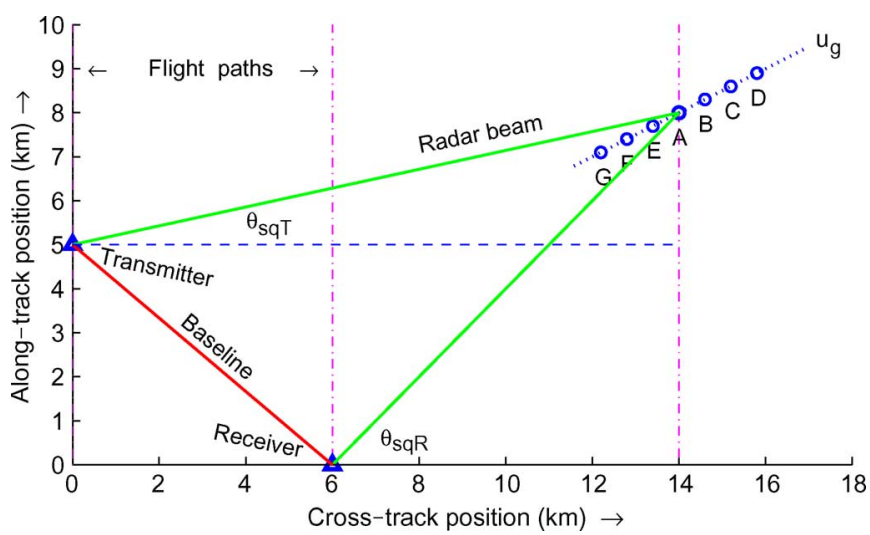

Fig. 4. Geometry of the bistatic simulation example (top view).

\section{A. Simulation Parameters}

The simulation uses the airborne SAR parameters given in Table I. An appreciable amount of antenna squint is assumed to introduce severe range/azimuth coupling. The oversampling ratio is 2.0 in range and 1.5 in azimuth. The range resolution is $2.05 \mathrm{~m}$ ( 2.09 cells), and the azimuth resolution is $1.48 \mathrm{~m}$, based on the definitions given in [22] and [23]. A Kaiser window with a smoothing coefficient of 2.5 is used to suppress the sidelobes in both range and azimuth.

Seven point targets are used in the simulation, as shown in Fig. 4. These point targets are illuminated at the same time by the composite bistatic beam. The separation between adjacent point targets is $200 \mathrm{~m}$ (exaggerated in the figure). All the point targets lie along a vector $\mathbf{u}_{g}$. This vector corresponds to the projection of the vector gradient of the bistatic range $\nabla R(\eta)$ onto the ground plane. Geometrically, $\nabla R(\eta)$ is a vector passing through the angular bisector of the bistatic angle $\beta$; it is used to define the processed resolution (refer to [22] and [23]).

The vector gradient $\nabla R(\eta)$ is given by

$$
\nabla R(\eta)=-\left(\hat{\mathbf{u}}_{t}+\hat{\mathbf{u}}_{r}\right)
$$

where $\hat{\mathbf{u}}_{t}$ is the unit vector from the point target to the transmitter, and $\hat{\mathbf{u}}_{r}$ is the unit vector from the point target to the receiver.

\section{B. Simulation Results}

The simulated compression results of the seven point targets are shown in Fig. 5. The SRC reference range is taken from the

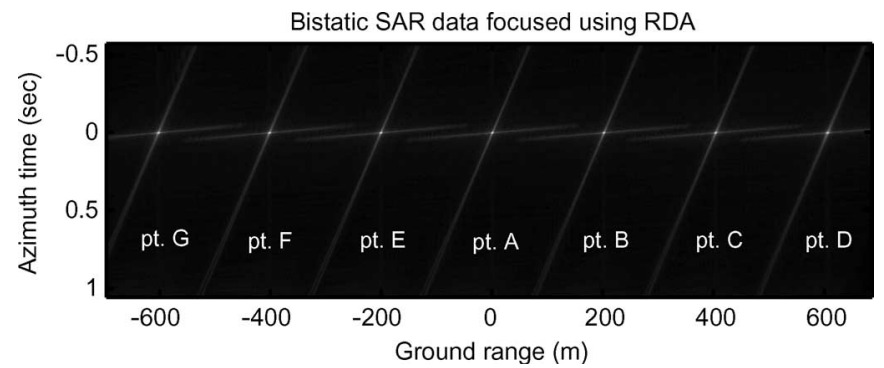

Fig. 5. Point targets focused using the bistatic RDA.
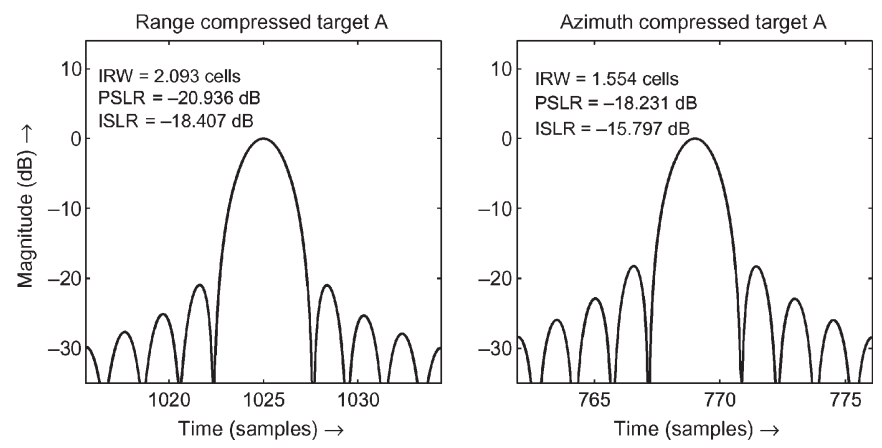

Fig. 6. Measurement of point target focus for point target A.
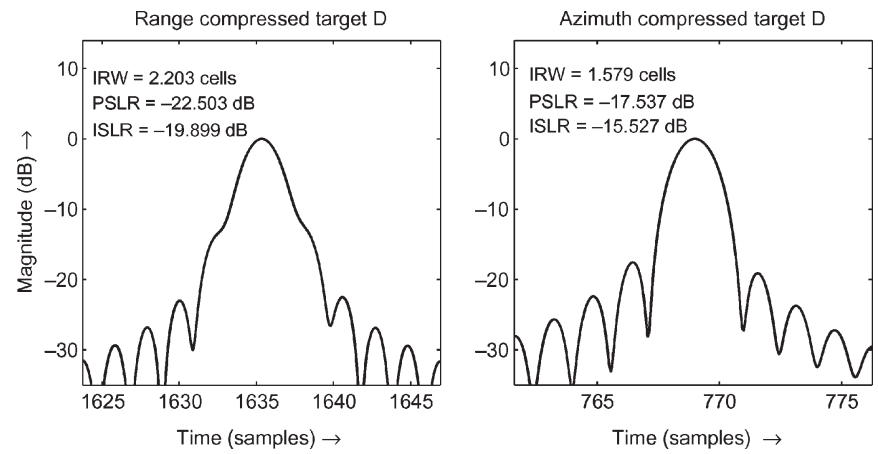

Fig. 7. Measurement of point target focus for point target D.

TABLE II

Point Target Quality Measurements

\begin{tabular}{|c|c|c|c|}
\hline Target & Broadening (\%) & PSLR (dB) & ISLR (dB) \\
\hline \hline \multicolumn{4}{|c|}{ Range Impulse Response } \\
\hline A & $<0.1$ & -20.9 & -18.4 \\
\hline B, E & $<0.1$ & $-19.5,-19.7$ & $-17.1,-17.4$ \\
\hline C, F & $1.70,1.68$ & $-19.0,-19.1$ & $-17.8,-17.9$ \\
\hline D, G & $5.02,4.99$ & $-22.5,-22.4$ & $-19.9,-19.7$ \\
\hline \hline \multicolumn{4}{|c|}{ Azimuth Impulse Response } \\
\hline A & $<0.1$ & -18.2 & -15.8 \\
\hline B, E & $<0.1$ & $-17.8,-17.9$ & $-15.6,-15.6$ \\
\hline C, F & $1.20,1.21$ & $-17.0,-17.3$ & $-15.0,-15.3$ \\
\hline D, G & $1.50,1.45$ & $-17.5,-17.5$ & $-15.5,-15.6$ \\
\hline
\end{tabular}

center target, i.e., Target A. The impulse responses of Target A and the edge target D are shown in Figs. 6 and 7, respectively, where the measured impulse response width (IRW) is annotated. The point target quality measurements for all the targets are given in Table II. It is seen that the reference target and its neighbors are well focused, but the edge targets are noticeably 

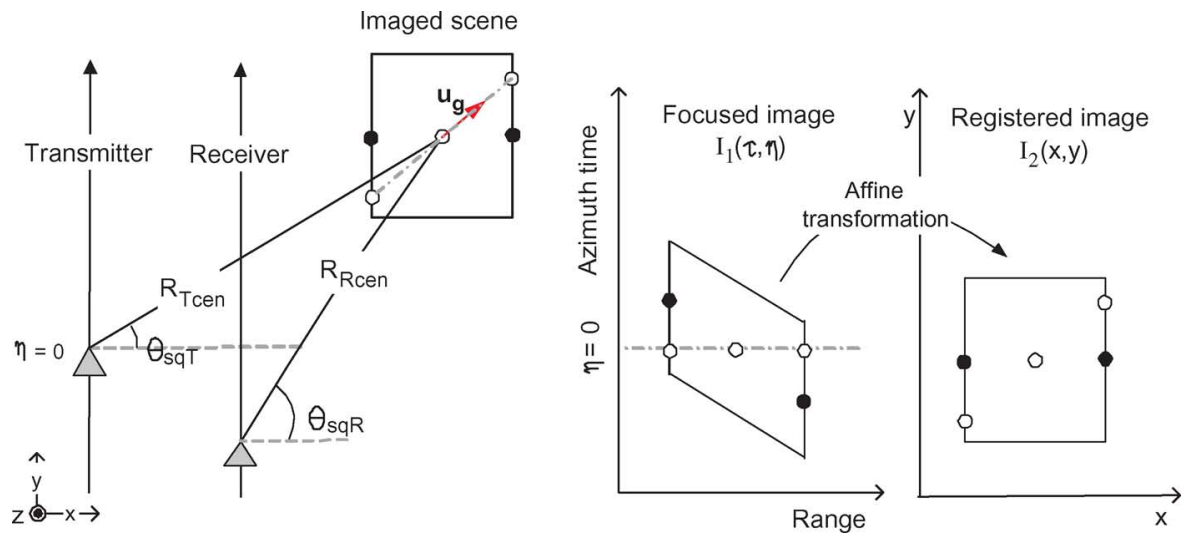

Fig. 8. Simple illustration to show how an imaged rectangular patch becomes a parallelogram in the focused map.

degraded. This degradation is due to the application of a rangeinvariant SRC filter for the whole scene.

If we restrict the quadratic phase error to be within $\pm \pi / 2$ at the ends of the range spectrum, the SRC filter can handle a range invariance region of $1270 \mathrm{~m}$ for this example. Targets $\mathrm{D}$ and $G$ are near the edges of the invariance region and have a phase error of $0.48 \pi$.

The theoretical peak sidelobe ratio (PSLR) and the integrated sidelobe ratio (ISLR) in range are -20.9 and $-18.5 \mathrm{~dB}$, respectively. In azimuth, the values are -18.4 and $-15.8 \mathrm{~dB}$. They are governed by the weighting used and by the azimuth beam pattern. The measured values for the edge targets are within $2 \mathrm{~dB}$ of the theoretical values.

\section{Implementation Issues}

To focus the imaged scene, the range derivatives $k_{1}, \ldots, k_{4}$ have to be determined as a function of range. Instead of calculating the derivatives for each range gate, a curve-fitting approach can be utilized. This will improve the computational efficiency of the algorithm. For instance, we calculate the range-varying derivatives for a representative set of targets equally spaced over a range invariance region. A curve is fitted for each derivative to obtain a polynomial function in range (a cubic polynomial was found to be sufficiently accurate for this 1.6-km range swath). Using these curves, we can then generate the required parameters for each range cell.

The image is focused in the bistatic range versus the azimuth time domain. The point targets are registered to their midaperture range and mid-aperture time. Fig. 8 illustrates how a rectangular patch becomes a parallelogram in the focused map. The three targets, which are denoted by white circles, lie along the same vector $\mathbf{u}_{g}$ and have the same beam center crossing time. When focused, they are registered at the same azimuth time. Thus, a rectangular patch on the ground plane appears as a parallelogram in the focused image, unless $\mathbf{u}_{g}$ is perpendicular to the flight path.

Image registration maps the focused image $I_{1}(\tau, \eta)$ to a flat earth plane $I_{2}(x, y)$, as shown in Fig. 8. The process involves two steps. First, the positions of known grid points in $I_{2}(x, y)$ are mapped onto $I_{1}(\tau, \eta)$. These grid points are usually chosen to be parallel and perpendicular to the flight path. Then, an affine transformation [24] is used to map the rest of the points.

\section{Approximate Bistatic RDA}

For coarser-resolution and lower-squint configurations, the range/azimuth coupling [which causes the "widening" of the energy observed in Fig. 2(b)] is less dependent on the azimuth frequency. In this case, we can neglect the azimuth frequency dependence when generating the SRC filter by using a coupling term that is fixed at the Doppler centroid frequency $f_{\eta_{c}}$. We can further simplify the derivation by neglecting the thirdorder and higher order terms of $f_{\tau}$ in the $\phi_{\text {src }}$ phase equation (10). Consequently, we can approximate the SRC phase by the quadratic function [19]

$$
\phi_{\mathrm{src}}\left(f_{\tau}, f_{\eta}\right) \approx \frac{\pi f_{\tau}^{2}}{K_{\mathrm{src}}}
$$

where the constant

$$
\begin{aligned}
\frac{1}{K_{\mathrm{src}}} \approx & \left\{\frac{c}{2 k_{2} f_{o}^{3}} f_{\eta_{c}}^{2}+\frac{3 k_{3}}{4 k_{2}^{3}}\left[\frac{k_{1} c}{f_{o}^{3}} f_{\eta_{c}}^{2}+\frac{c^{2}}{f_{o}^{4}} f_{\eta_{c}}^{3}\right]\right. \\
& \left.+\frac{9 k_{3}^{2}-4 k_{2} k_{4}}{16 k_{2}^{5}}\left[\frac{3 k_{1}^{2} c}{f_{o}^{3}} f_{\eta_{c}}^{2}+\frac{6 k_{1} c^{2}}{f_{o}^{4}} f_{\eta_{c}}^{3}+\frac{3 c^{3}}{f_{o}^{5}} f_{\eta_{c}}^{4}\right]\right\} .
\end{aligned}
$$

We can then combine the phase modulation of the range/ azimuth coupling and the range FM modulation together to form a new FM rate $K_{m}$ [19] as

$$
\frac{1}{K_{m}}=\frac{1}{K_{r}}-\frac{1}{K_{\mathrm{src}}} .
$$

In this case, the SRC and range compression can be implemented at the same azimuth time-domain step, which results in a more efficient algorithm. The processing steps for this approximate bistatic RDA are illustrated in Fig. 9. The steps are similar to the conventional RDA (refer to SRC Option 3 in [19]).

$K_{\text {src }}$ and $K_{m}$ are weakly range dependent and can often be kept constant for the imaged scene. If the range swath is too wide, the scene can be subdivided into contiguous segments and processed in range invariance regions.

\section{SUMMARY}

The bistatic SAR data can be processed with the RDA in the same way as the monostatic SAR data, but the RCMC, 


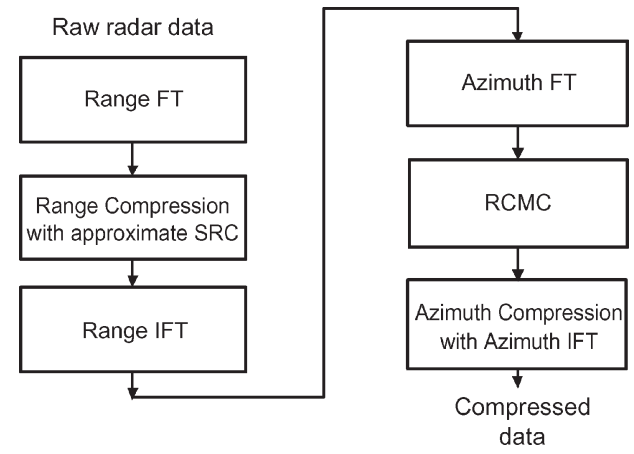

Fig. 9. Functional block diagram of approximate RDA.

SRC, and azimuth compression functions need to be modified accordingly.

To achieve this, a new analytical expression for the 2-D point target spectrum derived using series reversion is used for the RCMC, SRC, and azimuth compression parameters. The arbitrary accuracy of the spectrum can be achieved by retaining sufficient terms in the series reversion. The new spectrum applies to azimuth-invariant cases where the transmitter and receiver are operating in parallel tracks with the same velocity. A mild amount of azimuth variance can be accommodated using short azimuth block sizes.

The algorithm has been successfully tested with targets placed at different ranges. Although the RDA is used in the example, the approach to computing the bistatic spectrum can also be applied to the chirp scaling and $\omega-k$ algorithms.

\section{ACKNOWLEDGMENT}

The authors would like to thank DSO National Laboratories, Singapore, for providing scholarship funding for Mr. Neo and the National University of Singapore for encouraging Dr. Wong to study the interesting field of bistatic SAR processing.

\section{REFERENCES}

[1] P. Dubois-Fernandez, O. Ruault du Plessis, M. Wendler, R. Horn, G. Krieger, B. Vaizan, H. Cantalloube, D. Heuzé, and B. Gabler, "The ONERA-DLR bistatic experiment: Design of the experiment and preliminary results," in Proc. Adv. SAR Workshop, Jun. 25-27, 2003.

[2] I. Walterscheid, A. Brenner, and J. Ender, "Geometry and system aspects for a bistatic airborne SAR-experiment," in Proc. EUSAR Conf., Ulm, Germany, May 2004, pp. 567-570.

[3] C. Wu, "A digital system to produce imagery from SAR data," in Proc. AIAA Conf.: Syst. Des. Driven Sens., Oct. 1976.

[4] J. R. Bennett and I. G. Cumming, "A digital processor for the production of SEASAT synthetic aperture radar imagery," in Proc. SURGE Workshop, ESA Pub. SP-154, Frascati, Italy, Jul. 16-18, 1979.

[5] C. Wu, K. Y. Liu, and M. J. Jin, "Modeling and a correlation algorithm for spaceborne SAR signals," IEEE Trans. Aerosp. Electron. Syst., vol. AES-18, no. 5, pp. 563-575, Sep. 1982.

[6] R. K. Raney, H. Runge, R. Bamler, I. G. Cumming, and F. H. Wong, "Precision SAR processing using chirp scaling," IEEE Trans. Geosci. Remote Sens., vol. 32, no. 4, pp. 786-799, Jul. 1994.

[7] D. D'Aria, A. Monti Guarnieri, and F. Rocca, "Focusing bistatic synthetic aperture radar using dip move out," IEEE Trans. Geosci. Remote Sens., vol. 42, no. 7, pp. 1362-1376, Jul. 2004.

[8] A. Monti Guarnieri and F. Rocca, "Reduction to monostatic focusing of bistatic or motion uncompensated SAR surveys," Proc. Inst. Electr. Eng.-Radar, Sonar Navig., vol. 153, no. 3, pp. 199-207, Jun. 2006.

[9] O. Loffeld, H. Nies, V. Peters, and S. Knedlik, "Models and useful relations for bistatic SAR processing," IEEE Trans. Geosci. Remote Sens., vol. 42, no. 10, pp. 2031-2038, Oct. 2004.
[10] I. Walterscheid, J. H. G. Ender, A. R. Brenner, and O. Loffeld, "Bistatic SAR processing using an $\omega-k$ type algorithm," in Proc. IGARSS, Seoul, Korea, Aug. 2005, pp. 1064-1067.

[11] V. Giroux, H. Cantalloube, and F. Daout, "An Omega-K algorithm for SAR bistatic systems," in Proc. IGARSS, Seoul, Korea, Aug. 2005, pp. 1060-1063.

[12] R. Bamler and E. Boerner, "On the use of numerically computed transfer functions for processing of data from bistatic SARs and high squint orbital SARs," in Proc. IGARSS, Seoul, Korea, Jul. 2005, vol. 2, pp. 1051-1055.

[13] D. Hale, "Dip-moveout by Fourier transform," Geophysics, vol. 49, no. 14, pp. 741-757, Jun. 1984

[14] K. Natroshvili, O. Loffeld, and H. Nies, "Focusing of arbitrary bistatic SAR configurations," in Proc. EUSAR, Dresden, Germany, May 2006.

[15] O. Loffeld, K. Natroshvili, H. Nies, U. Gebhardt, S. Knedllik, A. Medrano-Ortiz, and A. Amankwah, "2D-scaled inverse Fourier transformation for bistatic SAR," in Proc. EUSAR, Dresden, Germany, May 2006.

[16] M. Rodríguez-Cassolá, G. Krieger, and M. Wendler, "Azimuth-invariant, bistatic airborne SAR processing strategies based on monostatic algorithms," in Proc. IGARSS, Seoul, Korea, Aug. 2005, pp. 1047-1050.

[17] Y. Neo, F. Wong, and I. G. Cumming, "A two-dimensional spectrum for bistatic SAR processing using series reversion," IEEE Geosci. Remote Sens. Lett., vol. 4, no. 1, pp. 93-96, Jan. 2007.

[18] M. Y. Jin and C. Wu, "A SAR correlation algorithm which accommodates large-range migration," IEEE Trans. Geosci. Remote Sens., vol. GRS-22, no. 6, pp. 592-597, Nov. 1984.

[19] I. G. Cumming and F. H. Wong, Digital Processing of Synthetic Aperture Radar Data: Algorithms and Implementation. Norwood, MA: Artech House, 2005.

[20] K. Eldhuset, "A new fourth-order processing algorithm for spaceborne SAR," IEEE Trans. Aerosp. Electron. Syst., vol. 34, no. 3, pp. 824-835, Jul. 1998.

[21] H. Zhong and X. Liu, "A fourth-order imaging algorithm for spaceborne bistatic SAR," in Proc. IGARSS, Denver, CO, Aug. 2006, pp. 1196-1199.

[22] G. P. Cardillo, "On the use of the gradient to determine bistatic SAR resolution," in Proc. Antennas Propag. Soc. Int. Symp., May 1990, vol. 2, pp. 1032-1035.

[23] I. Walterscheid, A. R. Brenner, and J. H. G. Ender, "Results on bistatic synthetic aperture radar," Electron. Lett., vol. 40, no. 19, pp. 1224-1225, Sep. 2004.

[24] H. T. Croft, K. J. Falconer, and R. K. Guy, Unsolved Problems in Geometry. New York: Springer-Verlag, 1991.

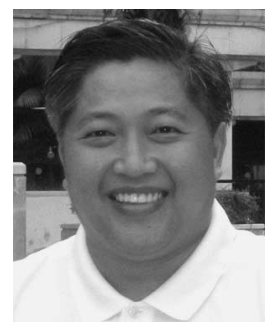

Yew Lam Neo received the B.Eng. degree in electrical engineering from the National University of Singapore, Singapore, in 1994 and the Ph.D. degree from the University of British Columbia, Vancouver, BC, Canada, in 2007.

From 1994 to 2001, he was an Engineer with DSO National Laboratories, Singapore, where he worked on SAR systems and software for real-time embedded systems. In 2006, he was a Visiting Scientist for three months with ZESS, University of Siegen, Siegen, Germany. He is currently with the Sensor Division, Radar Techniques Laboratory, DSO National Laboratories, where he is continuing his research in bistatic SAR signal processing.

Dr. Neo was awarded the Engineering Book Prize and was the winner of the Innovation Prize Award for his final year project work on a VHF frequency synthesizer. 


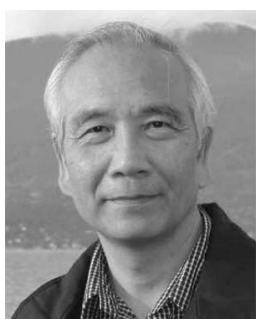

Frank H. Wong received the B.Eng. degree in electrical engineering from McGill University, Montreal, QC, Canada, the M.Sc. degree in electrical engineering from Queen's University, Kingston, ON, Canada, and the $\mathrm{Ph} . \mathrm{D}$. degree in computer science from the University of British Columbia (UBC), Vancouver, $\mathrm{BC}$, Canada.

Since 1977, he has been with MacDonald Dettwiler and Associates, Richmond, BC, where he worked in the Landsat and SPOT imaging area for the first few years. Then, his interest switched to SAR, and he has been working on airborne and satellite SAR processing and Doppler estimation ever since. He is affiliated with the Radar Remote Sensing Group, UBC, where he has also been a Sessional Lecturer on image processing for 18 years. He was a visitor to the National University of Singapore, Singapore, for a year in 1999, where he did research in bistatic SAR processing.

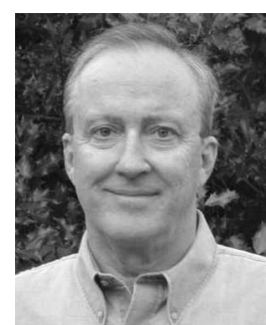

Ian G. Cumming (S'63-M'66-SM'05-LS'06) received the B.Sc. degree in engineering physics from the University of Toronto, Toronto, ON, Canada, in 1961, and the Ph.D. degree in computing and automation from Imperial College, University of London, London, U.K., in 1968.

In 1977, he was with MacDonald Dettwiler and Associates, Richmond, BC, Canada, where he developed SAR signal processing algorithms, including Doppler estimation and autofocus routines. He has been involved in the algorithm design of digital SAR processors for SEASAT, SIR-B, ERS-1/2, J-ERS-1, and RADARSAT, as well as several airborne radar systems. He was a Visiting Scientist with the German Aerospace Center, DLR, Oberpfaffenhofen, Germany, for one year in 1999. Since 1993, he has been with the Department of Electrical and Computer Engineering, University of British Columbia, Vancouver, BC, where he is the MacDonald Dettwiler/NSERC Industrial Research Chair in radar remote sensing. The Radar Remote Sensing Laboratory has published papers in the fields of SAR processing, SAR data encoding, satellite SAR two-pass interferometry, airborne along-track interferometry, polarimetric radar image classification, and SAR Doppler estimation. 